\title{
THOMAS-REICHE-KUHN SUM RULE FOR ELECTRON TRANSITIONS IN SOLIDS
}

\author{
S. OlszeWSKI \\ Institute of Physical Chemistry, Polish Academy of Sciences \\ Kasprzaka 44/52, 01-224 Warsaw, Poland \\ (Received January 26, 1998; revised version March 30, 1998; \\ in final form July 9, 1998)
}

\begin{abstract}
A well-known sum rule obtained for electron transitions between the atomic states by Thomas, Reiche and Kuhn (TRK) is examined for the case of transitions between delocalized electron states in cubic lattices. A characteristic point of the original TRK sum rule for the atomic states was its lack of dependence on the initial state of transitions. This situation holds also for the free-electron states in a metal but is changed in the case of electrons influenced by the presence of the field of the crystal core. Corrections to the original TRK result can be represented as a function of the quantum parameter labelling the initial electron state. Other moments of the spectral distribution function than those leading to the TRK sum rule have been calculated. A comparison of the relations found between different spectral moments for solids with similar relations obtained by Traini for the spectral moments of the atomic states has been done.
\end{abstract}

PACS numbers: $78.20 . \mathrm{Bh}$

\section{Introduction}

For the Bloch states describing electrons in perfect crystals there exists a strong selection rule which allows for electron dipole transitions to be done between the electron states belonging to different electron bands. Simultaneously, the rule forbids similar transitions between the states lying within the same band. This situation makes transitions in a solid formally similar to that observed in an atom because of often finite, and rather large, energy distances between the bands. At the same time, the situation contradicts the well-known property of the allowed low-energy transitions between the free-electron states in a solid which are obtained when, instead of Bloch's boundary conditions, the states satisfy the condition of vanishing at the crystal boundary. In the last case the electron states are represented by the standing-like wave functions. Similar low-energy transitions between electron levels should occur also in the case when the periodic potential 
of a perfect crystal lattice is considered, but the Bloch wave functions are replaced by the standing-like wave functions. Such a calculation of the band states can be done in the framework of the linear combination of atomic orbitals (LCAO) approximation, and consequences of the allowed dipole transitions obtained within one band can be examined.

In the present paper we demonstrate how the well-known Thomas-ReicheKuhn (TRK) sum rule for the dipole transitions between the electron states in an atom (Sec. 2) is modified in the case when electrons belong to one $s$-like electron band in a solid. First, in Sec. 3, we discuss in some detail the problem of the boundary conditions and their influence on the dipole transitions in a solid, whereas the tight-binding formalism suitable for calculations performed for a special case of solids having cubic lattices is presented in Sec. 4. The deviations from the TRK sum rule (Sec. 5) obtained on the basis of the tight-binding approximation of Sec. 4 originate not solely from the change of the electron mass at the band limit, but are due also to corrections dependent on the values of the quantum parameter labelling the electron states.

The TRK sum rule for an atom represents in fact only the first moment of the electric-dipole strength distribution function of that atom. However, other moments than the first one can be calculated for atoms and a corresponding calculation of the moments is presented in Sec. 6 for the case of the free-electron spectrum assumed for the electron states in a solid; the states belonging to that spectrum are extended throughout a large spherical block. In Sec. 7 the moments with a negative index obtained in Sec. 6 are discussed from the point of view of the influence which can be exerted on the free-electron results by a crystal lattice.

\section{Sum rules for electron transitions between the atomic quantum states}

Transition probabilities for an idealized one-electron problem, in which we merely deal with a single electron in a central field of an atomic core and neglect interactions with other electrons except for those described by the core, lead to some important general theorems represented by the so-called sum rules, see e.g. $[1-3]$.

Perhaps the best known sum rule in the atomic theory is the TRK sum rule [4-6]. This rule relates the dipole terms $z_{q i}$ between the quantum levels $|q\rangle$ and $|i\rangle$ which are coupled by the electric potential field directed along $z$-axis, namely

$$
z_{q i}=\langle q|z| i\rangle
$$

with the energy differences of the states $|q\rangle$ and $|i\rangle$ given by

$$
E_{q}-E_{i}=\hbar \omega_{q i}
$$

Let $|i\rangle$ be an initial level and $|q\rangle$ is a finite level. The sum rule is represented by the following formula:

$$
\frac{2 m_{\mathrm{e}}}{\hbar^{2}} \sum_{q}\left(E_{q}-E_{i}\right)|\langle q|z| i\rangle|^{2} \equiv \sum_{q} f_{q i}=1 .
$$


Energies $E_{i}$ and $E_{q}$ are the eigenvalues of the atomic Hamiltonian $H^{\mathrm{a}}$, the terms $f_{q i}$ are called the oscillator strengths. The summation runs over the complete set of the energy eigenstates [4-6].

The rule is important in the atomic, or molecular, optics. The so-called response function - or the electric-dipole strength distribution function - of the system is

$$
R^{\mathrm{D}}(\omega)=e^{2} \sum_{q=0}^{\infty}|\langle q|z| i\rangle|^{2} \delta\left(\omega-\omega_{q i}\right)
$$

where $\hbar \omega$ is the energy transferred to the system. If the state $|q\rangle$ is in the continuum, the sum must be replaced by an integral together with a substitution of an appropriate density of states, see e.g. $[7,8]$. The polarization vector of the external field is applied in Eq. (4) in the $z$-direction.

Formula (4) describes the strength distribution function of the system exposed to an external radiation of frequency $\omega$ in the long-wavelength approximation. This approximation means that the linear dimensions of the system are assumed to be small in comparison with the wavelength of the incident light, so solely the dipole terms (1) enter the strength distribution function (4). The energy conservation in Eq. (4) is represented by the delta-function factor. The spectral distribution of the atomic oscillator strengths has been reviewed, some time ago in Ref. [9]. More recently the problem of the TRK sum rule for a many-particle, atom has been raised in Ref. [10]. Parallel, the moments of the oscillator strength distributions of the order higher than one which is represented by the TRK sum rule have been calculated $[8,11]$. This derivation of the sum rules of different kinds appeared useful in the calculation of the particle excitations in the Bose superfluids [12] and also became important for the static response function for longitudinal and transverse excitations in the superfluid helium [13].

A direct application of the TRK sum rule comes into play when the total photoabsorption cross-section $\sigma^{\mathrm{D}}(\omega)$ is calculated in the long wavelength approximation

$$
\sigma^{\mathrm{D}}(\omega)=\frac{4 \pi^{2}}{\hbar c} \omega R^{\mathrm{D}}(\omega)=\frac{4 \pi^{2} e^{2}}{\hbar c} \omega \sum_{q=0}^{\infty}|\langle q|z| i\rangle|^{2} \delta\left(\omega-\omega_{q i}\right) .
$$

The total photoabsorption cross-section (5) integrated over the whole spectrum of frequencies $\omega$ gives

$$
\sigma^{\text {Dtot }}=\frac{4 \pi^{2} e^{2}}{\hbar c} \sum_{q=0}^{\infty} \omega_{q i}|\langle q|z| i\rangle|^{2} .
$$

Taking into account (2), expression (6) - with the accuracy to a constant multiplier - is identical to the left-hand side of Eq. (3). Formula (6) can be next transformed with the aid of the closure property for the eigenstates. We obtain

$$
\begin{aligned}
\sigma^{\mathrm{Dtot}} & =\frac{4 \pi^{2} e^{2}}{\hbar^{2} c} \frac{1}{2}\left\langle i\left|\left[z,\left[\widehat{H}^{\mathrm{a}}, z\right]\right]\right| i\right\rangle=\frac{4 \pi^{2} e^{2}}{\hbar^{2} c} \frac{1}{2}\left\langle i\left|\left[z,\left[\frac{\widehat{p}_{z}^{2}}{2 m_{\mathrm{e}}}, z\right]\right]\right| i\right\rangle \\
& =\frac{4 \pi^{2} e^{2}}{\hbar^{2} c} \frac{\hbar^{2}}{2 m_{\mathrm{e}}}
\end{aligned}
$$


because $z$ commutes with the position-coordinate dependent part of $\hat{H}^{\text {a }}$, as well as the components of the kinetic energy operator different than $\left(2 m_{\mathrm{e}}\right)^{-1} \widehat{p}_{z}^{2}$. This proves the right-hand side of Eq. (3) on condition that a constant multiplier of $4 \pi^{2} e^{2} / \hbar^{2} c$ has been omitted in Eqs. (6) and (7).

\section{Electron transitions between quantum states in crystals and the problem of the boundary conditions}

Expressions (1)-(6) represent essentially the one-electron approximation of the absorption problem for atoms. This approximation is very useful also for solids, where the amount of electrons is usually a huge number. In fact, the fundamentals of the theory of solids, especially the band theory, are based on the one-electron approximation.

However, the problem of the boundary conditions is essential in considering the optical transitions in solids. In the most part of considered cases the electrons are described by the Bloch wave functions which, in the case of a reduced influence of the crystal core, can be represented by the free-electron plane waves exp(i $k \cdot r)$. If electrons forming a gas in a metal sample are illuminated by the electromagnetic radiation, the photon momenta $\hbar q$, which can be acquired by electrons, should satisfy the relation

$$
k^{\prime}=k+q
$$

here $k$ and $k^{\prime}$ represent the electron states respectively before and after the photon absorption. Since the $q$ vector is usually (see e.g. [14]) by several orders of its magnitude less than the dimensions of the Brillouin zone, we have practically

$$
q \cong 0
$$

and obtain the conservation of the $k$ vector represented by

$$
k \cong k^{\prime} \text {. }
$$

This implies that there is no direct electron transition due to the electromagnetic absorption which should take place only between different electron bands. In consequence, in the Bloch picture, any of the electron transitions between energy levels in a metal should be accompanied by the corresponding input of the photon energy specified by the energy difference between two electron bands. In effect, for a perfect metal, represented by a constant core potential in the field of which the electrons move, there is practically no possibility of the transition of an individual electron due to the photon absorption.

A fully different situation exists when the metal is described by the standing free-electron wave functions. If we take a one-dimensional metal of length $L$, the wave functions are given by

$$
|q\rangle \equiv \psi_{q}(z)=\left(\frac{2}{L}\right)^{1 / 2} \sin \left(\frac{q \pi}{L} z\right)
$$

where the sinus form of the wave function comes from the boundary conditions

$$
\psi_{q}(0)=\psi_{q}(L)=0
$$


satisfied for any quantum state $|q\rangle$. Furthermore, if the electric potential field is directed along the metal, the matrix element coupling state $|q\rangle$ with some state $|i\rangle$ [see Eq. (1)] becomes

$$
\begin{gathered}
\langle q|z| i\rangle=\left(\frac{2}{L}\right) \int_{0}^{L} \sin \left(\frac{q \pi}{L} z\right) z \sin \left(\frac{i \pi}{L} z\right) \mathrm{d} z \\
=\frac{2}{\pi^{2}} L\left[(-1)^{q+i}-1\right] \frac{2 q i}{\left(q^{2}-i^{2}\right)^{2}} .
\end{gathered}
$$

Expression (13) does not vanish for any pair of states $|q\rangle$ and $|i\rangle$ having different parities, including also the neighbouring indices $q$ and $i$. A similar result will be applied to a three-dimensional electron gas. In this case the matrix elements will be equal to (13) on condition that the $x, y$-dependent part of the wave function is the same for states $|q\rangle$ and $|i\rangle$. In this way electron transitions can take place evidently between the states within the band providing us with a totally different pattern of the electron transitions than that obtained in the case of the (approximate) treatment of the Bloch states. In fact, all possible electron transitions within the band should be taken into account in the course of the derivation of the TRK sum rule for that band. This is done in Sec. 5. Evidently, if the dipole transitions (1) would be zero, the same result would be applied to the sum expression given in Eq. (3).

A clear presentation of the idea we have in mind can be done by extending the outline of the optical properties of metals given in Ref. [15]. The well-known dependence of the dielectric function of a free-electron metal on the frequency $\omega$ is

$$
\varepsilon(\omega)=1+\frac{4 \pi N e^{2}}{m_{\mathrm{e}}} \sum_{q} \frac{f_{q i}}{\omega_{q i}^{2}-\omega^{2}},
$$

where $N$ is the electron number in the unit of volume, the oscillator strength $f_{q i}$ is defined in Eq. (3), $\omega_{q i}$ is the frequency given in Eq. (2) where now $|q\rangle$ and $|i\rangle$ refer to the free-electron states in a metal. A usual approximation for a real metal is that $\omega_{q i}$ for transitions between $|q\rangle$ and $|i\rangle$, which give an important contribution to the sum presented in Eq. (14), are very small against $\omega$, so $\omega_{q i}$ can be neglected. Another step towards a real metal is that the free-electron mass $m_{e}$ should be replaced in Eqs. (3) and (14) by an effective mass $m_{\mathrm{eff}}$, an approximation which holds mainly for the nearly free-electron states, especially at the band limits. When $\varepsilon(\omega)$ is put equal to zero, Eq. (14) leads to the well-known expression for the plasmon frequency $\omega=\omega_{(\mathrm{p})}$ on condition that the sum rule given in Eq. (3) is satisfied also in the case of $m_{\mathrm{e}}$ replaced by $m_{\mathrm{eff}}$. Our point developed in the present paper is that not solely $m_{\mathrm{e}}$ should be changed into $m_{\mathrm{eff}}$ at the band limit, but a modification should concern also the sum rule representing the first moment of the spectral distribution function, namely

$$
m_{1}=\int \hbar \omega R^{\mathrm{D}}(\omega) \mathrm{d} \omega=\frac{\hbar^{2}}{2 m_{\mathrm{e}}} \sum_{q} f_{q i}=\frac{\hbar^{2}}{2 m_{\mathrm{e}}}
$$

itself [see (3)], here we have put $e=1$ for the sake of convenience. A very characteristic point in Eq. (3), or (3a), is that the final result for the sum of the oscillator 
strengths given there does not depend on the initial state $|i\rangle$. On the other hand, the modification of Eq: (3a) given below shows us that the corrective terms entering an extended formula for Eq. (3), or (3a), depend on the quantum parameter $\kappa$ labelling the initial state $|i\rangle$ (Sec. 5).

\section{Band methods suitable to calculation of the sum rules}

Among many methods which have been elaborated for calculating the band structure of solids one may deserve some special interest. This is the well-known LCAO method developed first by Bloch [16]. Our first aim is to examine the Thomas-Reiche-Kuhn rule within the framework of the LCAO method.

In this method the electron wave functions are combined from the atomic wave functions centred about the individual atoms. An especially simple description of the electron states is obtained when the LCAO approximation is limited to a single band of states based on one kind of the atomic wave functions $\varphi\left(r-\boldsymbol{R}_{\nu}\right)$, each $\varphi$ being centred about one atomic site $\boldsymbol{R}_{\nu}$ and the atoms are distributed in a regular lattice.

In a more accurate approach to the LCAO band theory we have the atomic orbitals $\varphi\left(\boldsymbol{r}-\boldsymbol{R}_{\nu}\right)$ replaced by the Wannier functions $a\left(\boldsymbol{r}-\boldsymbol{R}_{\nu}\right)$ which are centred about any site $\boldsymbol{R}_{\nu}$. In total, the one-electron wave function of a perfect crystal based on a single atomic state is

$$
\psi(r)=N_{A}^{-1 / 2} \sum_{\nu} A\left(\boldsymbol{R}_{\nu}\right) \varphi\left(\boldsymbol{r}-\boldsymbol{R}_{\nu}\right)
$$

where $N_{A}$ is the normalization coefficient.

Expressions for the coefficients $A\left(\boldsymbol{R}_{\nu}\right)$ of a perfect crystal depend on the boundary conditions imposed on the crystal wave functions (15). For the choice of the cyclic, or Born-von Karman, boundary conditions, the coefficients $A\left(\boldsymbol{R}_{\nu}\right)$ can be represented by a simple function of $\boldsymbol{R}_{\nu}$, namely

$$
A\left(\boldsymbol{R}_{\nu}\right)=\exp \left(\mathrm{i} k \cdot \boldsymbol{R}_{\nu}\right),
$$

here $k$ is the wave vector labelling the electron states distributed within the Brillouin zone. Difficulties connected with the calculation of the matrix element with the aid of Eqs. (15) and (16) are similar to those met for the matrix elements between the Bloch functions discussed at the end of Sec. 3. However, the coefficient functions other than those of the form given in Eq. (16) can be derived; they are useful, for example, in the case when the crystal wave functions vanish at some definite surface representing the boundary of a finite crystal block. Assuming that $\varphi\left(\boldsymbol{r}-\boldsymbol{R}_{\nu}\right)$ have a spherical symmetry about any $\boldsymbol{R}_{\nu}$, so

$$
\varphi\left(r-R_{\nu}\right)=\varphi_{s}\left(r-\boldsymbol{R}_{\nu}\right)
$$

is the atomic orbital of kind $s$, the coefficient function $A\left(\boldsymbol{R}_{\nu}\right)$ should transform according to the symmetry species compatible with the symmetry properties possessed by the crystal block. For example, for a block having the shape of a sphere and its centre is located in one of the crystal sites, the $A\left(\boldsymbol{R}_{\nu}\right)$ should transform according to the symmetry species of the crystal point group, on condition that the crystal transforms according to a symmorphic space group, so the crystal point group is a subgroup of the space group. In a special case of the cubic symmetry of 
the crystal point group taken as an example, the $A\left(\boldsymbol{R}_{\nu}\right)$ are the basis functions of the irreducible symmetry representations of a cube. In result, the quantum states classified according to the point-group symmetry species are represented by $A\left(\boldsymbol{R}_{\nu}\right)$ labelled by different parameters than the components of the vector $k$.

If $\widehat{H}^{\mathrm{c}}$ is an effective one-electron Hamiltonian acting on the crystal electrons which are described by Eqs. (15) and (16), the electron energy becomes

$$
E^{\mathrm{c}}=\int \psi^{*}(\boldsymbol{r}) \hat{H}^{\mathrm{c}} \psi(r) \mathrm{d} \boldsymbol{r}=\mathcal{E}^{\mathrm{B}}(k)
$$

The superscript B in the last term in Eq. (18) denotes the Bloch energy. Evidently, the second expression in Eq. (18) holds only for a special choice of $A\left(\boldsymbol{R}_{\nu}\right)$ done according to formula (16).

The simple and very well known expressions for $\mathcal{E}^{\mathrm{B}}(k)$ are obtained when the overlap between the atomic orbitals as well as the constant components in $\mathcal{E}^{\mathrm{B}}(k)$ are neglected and only an interaction between orbitals belonging to the nearest atomic neighbours is considered in the energy expression (18). This is the so-called tight-binding approximation of the LCAO method. For example, taking into account solely the orbitals having a spherical symmetry we obtain for simple-cubic (sc), face-centred cubic (fcc), and body-centred cubic (bcc) lattice, respectively (see e.g. [17])

$$
\begin{aligned}
& \mathcal{E}^{\mathrm{B}}(k)=-2 \beta^{\mathrm{sc}}\left(\cos k_{x}+\cos k_{y}+\cos k_{z}\right), \\
& \mathcal{E}^{\mathrm{B}}(k)=-4 \beta^{\mathrm{fcc}}\left(\cos k_{x} \cos k_{y}+\cos k_{y} \cos k_{z}+\cos k_{z} \cos k_{x}\right), \\
& \mathcal{E}^{\mathrm{B}}(k)=-8 \beta^{\mathrm{bcc}}\left(\cos k_{x} \cos k_{y} \cos k_{z}\right) .
\end{aligned}
$$

The length of the lattice parameter has been put equal to 1 and parameters $\beta^{\text {latt }}$ are the so-called interaction integrals between the nearest atomic neighbours characteristic of a given lattice [17].

An important point about Eq. (18) is that $\mathcal{E}^{\mathrm{B}}(k)$ can be transformed into an operator formula by the substitution

$$
k_{x} \rightarrow-\mathrm{i} \frac{\partial}{\partial X}=\widehat{p}_{X},
$$

and similar substitutions can be done for $k_{y}$ and $k_{z}$, so we obtain as the energy operator

$$
\widehat{W}=\mathcal{E}^{\mathrm{B}}(-\mathrm{i} \nabla)=\mathcal{E}^{\mathrm{B}}(\widehat{\boldsymbol{p}})
$$

for electrons moving in a perfect crystal described by the LCAO wave functions; $\widehat{p}_{X}, \widehat{p}_{Y}, \widehat{p}_{Z}$ are the momentum operators defined in the space of the components $X, Y, Z$ of the lattice site position vector $\boldsymbol{R}$; for the sake of brevity we have put the Planck constant $\hbar=1$.

The operator (21), introduced by Wannier and Slater [18-20], is especially useful when some perturbation of a perfect crystal lattice, done by a certain external potential, is considered. Another application of $\widehat{W}$ is to calculate the eigenfunctions $A\left(\boldsymbol{R}_{\nu}\right)$ and the corresponding eigenenergies $E^{\mathrm{c}}$ according to the formula

$$
\widehat{W} A\left(\boldsymbol{R}_{\nu}\right)=E^{c} A\left(\boldsymbol{R}_{\nu}\right)
$$

in the case when the boundary conditions of the crystal problem are different than those imposed by Born and von Karman. In fact, the differential operator $\widehat{W}$ 
entering Eq. (22) is not limited by the boundary conditions, because $\widehat{W}$ is based on the continuity equation when this equation is applied to the calculation of the coefficient values in different lattice sites beginning with the value of $A\left(\boldsymbol{R}_{\nu}\right)$ taken at some chosen site [21]. Details of the calculations of $A\left(\boldsymbol{R}_{\nu}\right)$ and $E^{\mathrm{c}}$ done, for example, for the electron wave functions in cubic lattices in the case when these functions satisfy the standing-like boundary conditions, are given in Refs. [21-26]. In the present paper we apply $\widehat{W}$ and $A\left(\boldsymbol{R}_{\nu}\right)$ to the calculation of the TRK sum rule for the band electrons.

Simple rules for the calculation of the matrix elements in the case of a perturbed $\widehat{W}$ have been developed in Ref. [27]. According to these rules any operator in the ordinary space $r$ of position coordinates, introduced as a perturbation of the original crystal Hamiltonian $\widehat{H}^{\mathrm{c}}$, should be transformed into the corresponding position coordinate of the space of the lattice vector $\boldsymbol{R}$ when this operator is introduced as a perturbation of $\widehat{W}$. Hence, the original perturbation $f(r)$ of $\widehat{H}^{c}$ becomes a perturbation $f(\boldsymbol{R})$ of $\widehat{W}$. In the case of the response function for photoabsorption done by electrons in a crystal, the energy components $E_{q}^{\mathrm{c}}$ and $E_{i}^{c}$ entering the difference

$$
\hbar \omega_{q i}^{c}=E_{q}^{c}-E_{i}^{c},
$$

instead of $\hbar \omega_{q i}$ in Eq. (4), can be represented as the eigenvalues of $\widehat{W}$ according to Eq. (22). Consequently, the component $z$ of the polarization vector in the ordinary space should be replaced by the component $Z$ in the space of the lattice vector. The closure property for the eigenstates of $\widehat{W}$ leads to the following formula for the photoabsorption cross-section integrated over the whole spectrum of frequencies $\omega$

$$
\begin{aligned}
& \int \sigma^{\mathrm{D}}(\omega) \mathrm{d} \omega=\frac{4 \pi^{2} e^{2}}{\hbar c} \int \omega \sum_{q=0}^{\infty}|\langle q|Z| i\rangle|^{2} \delta\left(\omega-\omega_{q i}^{\mathrm{c}}\right) \mathrm{d} \omega \\
& =\frac{4 \pi^{2} e^{2}}{\hbar c} \sum_{q=0}^{\infty} \omega_{q i}^{\mathrm{c}}|\langle q|Z| i\rangle|^{2}=\frac{4 \pi e^{2}}{\hbar^{2} c} \frac{1}{2}\langle i|[Z,[\widehat{W}, Z]]| i\rangle .
\end{aligned}
$$

This formula leads us to the TRK sum rule for electron transitions in crystal lattices; see Sec. 5. An important point is here, however, the problem of hermicity of the considered operators. The property of hermicity may depend not solely on the formula of the applied operators, but also on the form of the considered wave functions and the boundary conditions. The problem can be sound especially for solids where the Bloch wave functions for the crystal electrons are in a common use. In the case of the Bloch wave functions already an operator equal to a power of the coordinate variable, say $z$, is not a strictly Hermitian operator, see e.g. [28]. An explicit calculation performed on one-dimensional Bloch functions shows that the closure property is not satisfied already for the fourth power of the coordinate operator [29]. A better work of the closure property for the coordinate-dependent operators is obtained in the case of the standing-like free-electron wave functions taken instead of the Bloch wave functions. But also in the case of the standing-like wave functions the closure property ceases to be satisfied when the coordinate-dependent operators are combined with the momentum 
operators and the total power exponent of the combined operator is larger than 3 , see [29]. Difficulties in construction of the high-order Hermitian operators for solids are well known $[30,31]$. In fact, any operator can be strictly Hermitian only when the considered area is extended to infinity and the wave functions are represented by the appropriate class of the functions. This condition is fulfilled mainly for atoms, but usually it is not satisfied for solids. Hence, any result obtained with the use of the closure property for the solid states should be considered only as an approximate one, especially in the case of the operators combining high powers of momentum and position coordinates. Consequently, in our calculations we refrain from the moments of the strength distribution function (4) higher than the second one, see Sec. 6 . The importance of the boundary conditions in connection with the TRK sum rule has been pointed out in Ref. [32].

\section{Thomas-Reiche-Kuhn sum rule for the band electrons}

The commutation properties of the operator $\widehat{W}$ give $[27,33,34]$

$$
\begin{aligned}
& {[Z, \widehat{W}]=Z \widehat{W}-\widehat{W} Z=\mathrm{i} \frac{\partial \widehat{W}}{\partial \widehat{p}_{Z}},} \\
& {\left[Z, \frac{\partial \widehat{W}}{\partial \widehat{p}_{Z}}\right]=Z \frac{\partial \widehat{W}}{\partial \widehat{p}_{Z}}-\frac{\partial \widehat{W}}{\partial \widehat{p}_{Z}} Z=\mathrm{i} \frac{\partial^{2} \widehat{W}}{\partial \widehat{p}_{Z}^{2}},}
\end{aligned}
$$

etc., so the first moment of the spectral density (putting $e=1$ ) is

$$
\begin{aligned}
m_{1} & \left.=\frac{1}{2}\langle i|[Z, \widehat{W}, Z]\right]|i\rangle=\frac{1}{2}\left\langle i\left|\left[Z,-\mathrm{i} \frac{\partial \widehat{W}}{\partial \widehat{p}_{Z}}\right]\right| i\right\rangle \\
& =\frac{1}{2}(-\mathrm{i}) \mathrm{i}\left\langle i\left|\left[\frac{\partial^{2} \widehat{W}}{\partial \widehat{p}_{Z}^{2}}\right]\right| i\right\rangle=\frac{1}{2}\left\langle i\left|\frac{\partial^{2} \widehat{W}}{\partial \widehat{p}_{Z}^{2}}\right| i\right\rangle .
\end{aligned}
$$

Neglecting the constant components in $\mathcal{E}^{\mathrm{B}}(k)$ and $\widehat{W}$ and putting the length of the lattice parameter equal to unity, we obtain the following power expansions of $\widehat{W}$ :

$$
\begin{aligned}
\widehat{W}= & -6 \beta^{\mathrm{sc}}+\frac{2 \beta^{\mathrm{sc}}}{2 !}\left(\widehat{p}_{X}^{2}+\widehat{p}_{Y}^{2}+\widehat{p}_{Z}^{2}\right)-\frac{2 \beta^{\mathrm{sc}}}{4 !}\left(\widehat{p}_{X}^{4}+\widehat{p}_{Y}^{4}+\widehat{p}_{Z}^{4}\right)+\ldots, \\
\widehat{W}= & -12 \beta^{\mathrm{fcc}}+4 \beta^{\mathrm{fcc}}\left(\widehat{p}_{X}^{2}+\widehat{p}_{Y}^{2}+\widehat{p}_{Z}^{2}\right)-\frac{4 \beta^{\mathrm{fcc}}}{2 ! 2 !}\left(\widehat{p}_{X}^{2} \widehat{p}_{Y}^{2}+\widehat{p}_{Y}^{2} \widehat{p}_{Z}^{2}+\widehat{p}_{Z}^{2} \widehat{p}_{X}^{2}\right) \\
& -\frac{8 \beta^{\mathrm{fcc}}}{4 !}\left(\widehat{p}_{X}^{4}+\widehat{p}_{Y}^{4}+\widehat{p}_{Z}^{4}\right)+\ldots, \\
\widehat{W}= & -8 \beta^{\mathrm{bcc}}+\frac{8 \beta^{\mathrm{bcc}}}{2 !}\left(\widehat{p}_{X}^{2}+\widehat{p}_{Y}^{2}+\widehat{p}_{Z}^{2}\right)-\frac{8 \beta^{\mathrm{bcc}}}{2 ! 2 !}\left(\widehat{p}_{X}^{2} \widehat{p}_{Y}^{2}+\widehat{p}_{Y}^{2} \widehat{p}_{Z}^{2}+\widehat{p}_{Z}^{2} \widehat{p}_{X}^{2}\right) \\
& -\frac{8 \beta^{\mathrm{bcc}}}{4 !}\left(\widehat{p}_{X}^{4}+\widehat{p}_{Y}^{4}+\widehat{p}_{Z}^{4}\right)+\ldots
\end{aligned}
$$

for the sc, fcc and bcc lattice, respectively, because of Eqs. (19)-(19b), (20) and (21).

If we consider energies near the band limit represented by small $k_{x}, k_{y}$ and $k_{z}$ and retain only the second-order terms in the expansions of cosines entering 
Eqs. (19)-(19b), the $k$-dependent part of the energy $\mathcal{E}^{\mathrm{B}}(k)$ is proportional to $k^{2}$ just as in the case of free electrons. Hence, we may treat these electrons as free with the following effective masses:

$$
m_{\mathrm{eff}}^{\mathrm{scc}}=\frac{\hbar^{2}}{2 \beta^{\mathrm{sc}}}, \quad m_{\mathrm{eff}}^{\mathrm{fcc}}=\frac{\hbar^{2}}{8 \beta^{\mathrm{fcc}}}, \quad m_{\mathrm{eff}}^{\mathrm{bcc}}=\frac{\hbar^{2}}{8 \beta^{\mathrm{bcc}}},
$$

for the sc, fcc, and bcc lattice, respectively. From Eqs. (28)-(28b) and (29) we obtain for the same lattices

$$
\begin{aligned}
& \frac{\partial^{2} \widehat{W}}{\partial \widehat{p}_{Z}^{2}}=2 \beta^{\mathrm{sc}}\left(1-\frac{1}{2 !} \widehat{p}_{Z}^{2}+\ldots\right)=\frac{\hbar^{2}}{m_{\mathrm{eff}}^{\mathrm{sc}}}\left(1-\frac{1}{2 !} \widehat{p}_{Z}^{2}+\ldots\right), \\
& \frac{\partial^{2} \widehat{W}}{\partial \widehat{p}_{Z}^{2}}=\frac{\hbar^{2}}{m_{\mathrm{eff}}^{\mathrm{fcc}}}\left[1-\frac{1}{2} \widehat{p}_{Z}^{2}-\frac{1}{4}\left(\widehat{p}_{X}^{2}+\widehat{p}_{Y}^{2}\right)+\ldots\right], \\
& \frac{\partial^{2} \widehat{W}}{\partial \widehat{p}_{Z}^{2}}=\frac{\hbar^{2}}{m_{\mathrm{eff}}^{\text {bcc }}}\left[1-\frac{1}{2 !}\left(\widehat{p}_{X}^{2}+\widehat{p}_{Y}^{2}+\widehat{p}_{Z}^{2}\right)+\ldots\right]
\end{aligned}
$$

Operators $(30)-(30 \mathrm{~b})$ can be now substituted between the bra expressions representing the standing-like coefficient functions for cubic lattices, and similar ket expressions, viz.

$$
|i\rangle=\left|A^{\Gamma, \mu, \lambda}(\kappa, \boldsymbol{R})\right\rangle .
$$

Here $\Gamma$ labels the irreducible representation of the crystal point group, $\mu$ labels the row of that representation and $\lambda$ is the index labelling the solution of the eigenequation (22) obtained for a given $\Gamma$ and $\mu$, the quantum parameter $\kappa$ is obtained from the boundary condition.

In the case considered in the paper it is sufficient to take only one irreducible representation, viz. $\Gamma=\Gamma_{1}$, to calculations. This is so because the situation within a perfect crystal repeats itself in each lattice site, on condition that the crystal sites are located far enough from the crystal boundary. In particular, a site located at the origin $\mathbf{0}$ of the coordinate system can be taken to calculations as representing other sites of a crystal. In this case solutions (31) belonging only to $\Gamma_{1}$ are of importance because solely the solutions of this kind do not vanish at 0 (see [21, 22] and Sec. 7). We arrive at the expansions

$$
\begin{aligned}
m_{1}^{\mathrm{sc}} & =\frac{1}{2} \frac{\hbar^{2}}{m_{\mathrm{eff}}^{\mathrm{sc}}}\left\langle A^{\Gamma_{1}, \lambda}(\kappa, \boldsymbol{R})\left|1+\frac{1}{2} \frac{\partial^{2}}{\partial Z^{2}}+\ldots\right| A^{\Gamma_{1}, \lambda}(\kappa, \boldsymbol{R})\right\rangle \\
& =\frac{1}{2} \frac{\hbar^{2}}{m_{\mathrm{eff}}^{\mathrm{sc}}}\left(1-\frac{1}{2} \cos ^{2} \Theta \kappa^{2}+\ldots\right)=\frac{1}{2} \frac{\hbar^{2}}{m_{\mathrm{eff}}^{\mathrm{sc}}}\left(1-\frac{1}{6} \kappa^{2}+\ldots\right), \\
m_{1}^{\mathrm{fcc}} & =\frac{1}{2} \frac{\hbar^{2}}{m_{\mathrm{eff}}^{\mathrm{fcc}}}\left\langle A^{\Gamma_{1}, \lambda}(\kappa, \boldsymbol{R})\right| 1+\frac{1}{2} \frac{\partial^{2}}{\partial Z^{2}}-\frac{1}{4}\left(\frac{\partial^{2}}{\partial X^{2}}+\frac{\partial^{2}}{\partial Y^{2}}\right) \\
& +\ldots\left|A^{\Gamma_{1}, \lambda}(\kappa, \boldsymbol{R})\right\rangle=\frac{1}{2} \frac{\hbar^{2}}{m_{\mathrm{eff}}^{\mathrm{fcc}}}\left(1-\frac{1}{3} \kappa^{2}+\ldots\right),
\end{aligned}
$$




$$
\begin{aligned}
& m_{1}^{\mathrm{bcc}}=\frac{1}{2} \frac{\hbar^{2}}{m_{\mathrm{eff}}^{\mathrm{bcc}}}\left\langle A^{\Gamma_{1}, \lambda}(\kappa, \boldsymbol{R})\left|1-\frac{1}{2}\left(-\frac{\partial^{2}}{\partial X^{2}}-\frac{\partial^{2}}{\partial Y^{2}}-\frac{\partial^{2}}{\partial Z^{2}}\right)+\ldots\right|\right. \\
& \left.\quad \times A^{\Gamma_{1}, \lambda}(\kappa, \boldsymbol{R})\right\rangle=\frac{1}{2} \frac{\hbar^{2}}{m_{\mathrm{eff}}^{\mathrm{bcc}}}\left(1-\frac{1}{2} \kappa^{2}+\ldots\right),
\end{aligned}
$$

for the sc, fcc and bcc lattice, respectively; $\Theta$ is the angle between axis $Z$ and vector $\boldsymbol{R}$. The normalization property of $A^{\Gamma_{1}, \lambda}(\kappa, R)$ has been taken into account in the calculations together with the weighted averaging process over the angle $\Theta$, namely

$$
\begin{aligned}
& \int_{0}^{\pi} \sin ^{2} \Theta \sin \Theta \mathrm{d} \Theta\left(\int_{0}^{\pi} \sin \Theta \mathrm{d} \Theta\right)^{-1} \\
& \quad=2 \int_{0}^{\pi} \cos ^{2} \Theta \sin \Theta \mathrm{d} \Theta\left(\int_{0}^{\pi} \sin \Theta \mathrm{d} \Theta\right)^{-1}=\frac{2}{3} .
\end{aligned}
$$

For small $\kappa$ the results $(32)-(32 \mathrm{~b})$ depend solely on $\kappa^{2}$. This is so because any coefficient function given in the expression (31) is also an eigenfunction of the Laplacian operator with the eigenvalue equal to $\kappa^{2}$, see [21-25]. The dependence on $\lambda$ of the expressions (32)-(32b) can be exhibited when higher powers of $\kappa$ than the second one are taken into account. This dependence is connected with the fact that any of the expressions $A^{\Gamma_{1}, \lambda}(\kappa, R)$ has its eigenvalue $E^{\Gamma_{1}, \lambda}(\kappa)$, so for a large enough $\kappa$ we have different energies labelled by $\lambda$, see $[21,22]$ and Sec. 6 . The moments $m_{1}$ for the band electrons [see (32)-(32b)] differ from the nearly-free electron expression

$$
m_{1}=\frac{\hbar^{2}}{2 m_{\mathrm{eff}}^{\text {latt }}}
$$

by their dependence on the quantum parameter $\kappa$, the superscript latt in Eq. (27a) is an abbreviation of sc, fcc and bcc.

Let us note that expressions similar to (32)-(32b) can be deduced also on the basis of the LCAO wave functions having the itinerant coefficient functions (16). However, the calculation based on the standing-like coefficient functions (31) allows us to avoid the problem of divergence of the matrix elements for the electric field extended over the Bloch elementary domain (see e.g. [31]) as well as, to some degree, the problem of the lack of hermicity, see the end of Sec. 4. The expressions (32) $-(32 \mathrm{~b})$ depend on the radius $R_{\mathrm{d}}$ of the crystal block by the intermediate of the quantum parameter

$$
\kappa=\frac{n \pi}{R_{\mathrm{d}}}
$$

for the states building up the electron density at the site 0 (see e.g. [22]), $n$ is a positive integer number. 


\subsection{Plasmon problem in transition metals and semiconductors}

In the first step, let us note that the results obtained in Sec. 5 explain why no plasmon absorption can occur for tightly-bound electrons which are typical of, for example, transition metals. By averaging the operator entering the expressions (27) and $(30)-(30 \mathrm{~b})$ over the Bloch coefficient function, i.e.,

$$
m_{1}=\frac{1}{2}\left\langle\exp (\mathrm{i} k \cdot \boldsymbol{R})\left|\frac{\partial^{2} \widehat{W}}{\partial \widehat{p}_{Z}^{2}}\right| \exp (\mathrm{i} \boldsymbol{k} \cdot \boldsymbol{R})\right\rangle,
$$

we obtain

$$
\begin{aligned}
& m_{1}^{\mathrm{sc}}=\beta^{\mathrm{sc}} \cos k_{z}, \\
& m_{1}^{\mathrm{fcc}}=2 \beta^{\mathrm{fcc}} \cos k_{z}\left(\cos k_{x}+\cos k_{y}\right), \\
& m_{1}^{\mathrm{bcc}}=4 \beta^{\mathrm{bcc}} \cos k_{x} \cos k_{y} \cos k_{z} .
\end{aligned}
$$

For a given constant energy, say the Fermi energy, we have, in general very different values of $k_{x}, k_{y}$ and $k_{z}$ for. that energy because of the well-known anisotropy of the Fermi surface for the tightly-bound electrons. This property provides us with an oscillatory character of the expressions (34)-(34b) in the $k$ space, so its average calculated over the values of $k$ belonging to the Fermi surface should cancel. This situation should be repeated also, for example, for the tighthly-bound $d$ electrons which can provide us with similar trigonometric expressions for $m_{1}$ as those given in Eqs. (34)-(34b), see e.g. [35]. The states belonging to anisotropic surfaces of a constant energy predominate in the band of states of a transition metal, so the whole band is, in principle, not suitable to produce a plasmon.

A different situation may occur, however, for semiconductors having a considerable amount of carriers present in the conduction band, for example, due to the effect of donors. Usually, the concentration of electron carriers is much smaller than a concentration of electrons in a metal band, because the filling of the conduction band is small. However, these carriers can behave similarly to the tightly-bound electrons in a metal band near the band limit. This makes possible to apply the formulae (3), (14) and next (24) and (32)-(32b) also for the carriers, on condition that a constant multiplying $\kappa^{2}$ is replaced by another constant, characteristic of the band of a given semiconductor. Usually, such a constant does not exceed unity. Since the concentration of carriers is smaller by several orders than that of electrons, the size of $\kappa$ occupied within a conduction band should be much smaller than 1. Therefore, approximately, the correction to TRK rule introduced in the expressions (32)-(32b) should be also small. Recently, a conventional plasmon dispersion formula has been successfully applied to $n$-doped GaAs semiconductors having different carrier densities [36].

\section{Other moments than $m_{1}$ calculated for cubic lattices}

Beyond the moment $m_{1}$ calculated in Eq. (27) as well as in Eqs. (32)-(32b), also other moments of the spectral density can be considered, see e.g. [8]. Rather recently the moments other than $m_{1}$ have found their applications especially in the studies of the nuclear matter $[12,13]$. Because of inaccuracies connected with 
the application of the closure property to the crystal states (see the end of Sec. 3), the calculation of the higher moments done in the present paper is limited solely to $m_{2}$, but the moments having negative indices are also considered.

The moment $m_{2}$ of the spectral density is

$$
m_{2}=\int \mathrm{d} \omega(\hbar \omega)^{2} R^{\mathrm{D}}(\omega)=\frac{1}{2}\langle i|\{[Z, \widehat{W}],[\widehat{W}, Z]\}| i\rangle .
$$

We have put here $e=1$ for the electron charge, the curly brackets in Eq. (35) denote the anticommutator. Because of Eq. (25) we obtain

$$
m_{2}=\left\langle i\left|\left(\frac{\partial \widehat{W}}{\partial \widehat{p}_{Z}}\right)^{2}\right| i\right\rangle,
$$

in which

$$
\frac{\partial \widehat{W}}{\partial \widehat{p}_{Z}}=2 \beta^{\mathrm{sc}}\left(\widehat{p}_{Z}-\frac{1}{6} \widehat{p}_{Z}^{3}+\ldots\right)
$$

for the sc lattice and similar expressions can be obtained for the fcc and bcc lattices, respectively. When only the lowest powers of the momentum operator in Eq. (36) and similar operators for the fcc and bcc lattices as well as the coefficient function (31) for $\Gamma=\Gamma_{1}$ are taken into account, we obtain from (35)

$$
\begin{aligned}
& m_{2}^{\mathrm{sc}} \cong 4\left(\beta^{\mathrm{sc}}\right)^{2}\left\langle A^{\Gamma_{1}, \lambda}(\kappa, R)\left|-\frac{\partial^{2}}{\partial Z^{2}}\right| A^{\Gamma_{1}, \lambda}(\kappa, R)\right\rangle=\frac{4}{3}\left(\beta^{\mathrm{sc}}\right)^{2} \kappa^{2}, \\
& m_{2}^{\mathrm{fcc}}=\frac{64}{3}\left(\beta^{\mathrm{fcc}}\right)^{2} \kappa^{2} \\
& m_{2}^{\mathrm{bcc}}=\frac{64}{3}\left(\beta^{\mathrm{bcc}}\right)^{2} \kappa^{2} .
\end{aligned}
$$

In the calculation of the expressions (37)-(37b) the averaging process over the angle $\Theta$ [see Eqs. (32) and (32a)] has been taken into account.

The inverse energy-weighted sum rules may have interesting applications (see [8] and Sec. 6.1 of the present paper), so we consider also the moments $m_{\tau}$ having a negative $\tau$. For $\tau=-1,-2, \ldots$ we obtain the following equations:

$$
\begin{aligned}
& m_{-1}=\int \mathrm{d} \omega \frac{R^{\mathrm{D}}(\omega)}{\hbar \omega}=e^{2} \sum_{q \neq 0} \frac{|\langle q|z| i\rangle|^{2}}{E_{q}-E_{i}} \\
& m_{-2}=\int \mathrm{d} \omega \frac{R^{\mathrm{D}}(\omega)}{(\hbar \omega)^{2}}=e^{2} \sum_{q \neq 0} \frac{|\langle q|z| i\rangle|^{2}}{\left(E_{q}-E_{i}\right)^{2}}
\end{aligned}
$$

The constant $e$ has been recovered in Eqs. (38) and (38a).

A convenient approach to Eqs. (38), (38a), etc. for solids can be done in the framework of the free-electron approximation. In this approximation the standing electron wave functions are

$$
\psi_{n l m}=N_{n l m} j_{l}\left(\kappa_{n l} r\right) P_{l}^{m}(\cos \vartheta) \exp (\mathrm{i} m \varphi)=|\kappa l m\rangle
$$

since we assume that the electrons are enclosed in a spherical potential cavity having a finite radius $R_{\mathrm{d}}$; in consequence, coordinate $z$ in Eqs. (38) and (38a), 
as well as coordinate $r$ in Eq. (39), represent, respectively, the polarization vector and position coordinate in the ordinary space. The quantum parameters $\kappa_{n l}$ entering the argument of the spherical Bessel functions $j_{l}$ in Eq. (39) are obtained from the requirement of vanishing of Eq. (39) for any $n$ and $l$. The functions $P_{l}^{m}(\cos \vartheta) \exp (\operatorname{im} \varphi)$ represent spherical harmonics, $N_{n l m}$ is the normalization coefficient. A rather recent discussion of the problem of particles moving in a spherical cavity is given in Ref. [37].

According to the density-functional approach, the situation in the whole volume of the perfect metal can be analyzed in only one metal point, because due to a large number of electrons present in a metal - the electron density in one point, say $r=\mathbf{0}$, chosen within the metal sample repeats itself over the whole metal volume. This is so on condition that we neglect the regions close to the metal boundary as well as the influence of the crystal core. In this way the perturbation calculation can be limited to the wave functions

and

$$
\psi_{n 00}=N_{n 00} j_{0}\left(\kappa_{n 0} r\right)=N_{n 00} \frac{\sin \left(\kappa_{n 0} r\right)}{\kappa_{n 0} r}=|\kappa, 0,0\rangle
$$

$$
\psi_{n^{\prime} 10}=N_{n^{\prime} 10} j_{1}\left(\kappa_{n^{\prime} 1} r\right) P_{1}^{0}(\cos \vartheta)=\left|\kappa^{\prime}, 1,0\right\rangle,
$$

because solely the wave functions (40) are building up the electron density in a chosen metal point

$$
r=0
$$

and only the wave functions (41) can ke coupled with the wave functions (40) by the dipole polarization operator

$$
e z=e r \cos \vartheta=e r P_{1}^{0}(\cos \vartheta)
$$

for a thorough discussion of the perturbation approach outlined above see Ref. [38]. The normalization coefficients entering (40) and (41) are

$$
\begin{aligned}
N_{n 00} & =\frac{\kappa_{n 0}}{\left(2 \pi R_{\mathrm{d}}\right)^{1 / 2}}, \\
\therefore N_{n^{\prime} 10} & =\frac{3^{1 / 2} \kappa_{n^{\prime} 1}}{\left(2 \pi R_{\mathrm{d}}\right)^{1 / 2}} .
\end{aligned}
$$

The square value of the matrix element between Eqs. (40), (41) and (43) entering the nominator of $m_{-1}$ is

$$
e^{2}\left|\left\langle\kappa_{n 0}, 0,0|z| \kappa_{n^{\prime} 1}, 1,0\right\rangle\right|^{2}=e^{2} 3^{-1} R_{\mathrm{d}}^{-2}\left(\kappa_{n 0}-\kappa_{n^{\prime} 1}\right)^{-4},
$$

whereas the energy expression entering the denominator of $m_{-1}$ is

$$
E_{n 0}-E_{n^{\prime} 1}=\frac{\hbar^{2}}{2 m_{\mathrm{e}}}\left(\kappa_{n 0}^{2}-\kappa_{n^{\prime} 1}^{2}\right)
$$

see [38]. The quantum parameters entering Eqs. (44) and (45) are

$$
\begin{aligned}
& \kappa_{n 0}=\frac{n \pi}{R_{\mathrm{d}}}, \\
& \kappa_{n^{\prime} 1} \approx \frac{\pi}{R_{\mathrm{d}}}\left[\frac{1}{2}+n^{\prime}-\frac{2}{\pi\left(1+2 n^{\prime}\right)}\right],
\end{aligned}
$$


see [39] for a discussion of the expression (47). Since in the predominant number of the electron states considered in a metal the indices $n$ and $n^{\prime}$ are large numbers (let us consider, e.g., the electron excitations, near the Fermi level), the third component within the square brackets in the expression (47) can be neglected, so

$$
\begin{aligned}
& \kappa_{n 0}-\kappa_{n^{\prime} 1} \cong \frac{\pi}{R_{\mathrm{d}}}\left(n-\frac{1}{2}-n^{\prime}\right) \\
& \kappa_{n 0}^{2}-\kappa_{n^{\prime} 1}^{2} \cong\left(\frac{\pi}{R_{\mathrm{d}}}\right)^{2}\left(n-\frac{1}{2}-n^{\prime}\right)\left(n+\frac{1}{2}+n^{\prime}\right) .
\end{aligned}
$$

The formulae obtained above imply that an important contribution to $m_{-1}$, $m_{-2}$, etc., calculated for a chosen $n$ labelling the state (40) is given already by a few components of each sum entering (38) and (38a) which are taken for

$$
n^{\prime} \approx n \gg 1
$$

let us note that here the indices $n^{\prime}>n$, as well as $n^{\prime}<n$, should be considered. Beginning with $n^{\prime}=n$ and taking into account $n \gg 1$ we obtain from Eqs. (44) and (45) the following sum for $m_{-1}$ :

$$
m_{-1} \approx \frac{2^{3}}{n^{2}}\left(\frac{1}{1^{4}}+\frac{1}{3^{4}}+\frac{1}{5^{4}}+\ldots\right) \frac{1}{3} e^{2} R_{\mathrm{d}}^{4} \frac{2 m_{\mathrm{e}}}{\hbar^{2} \pi^{2}} .
$$

In a similar way, the sum for $m_{-2}$ is

$$
m_{-2} \approx \frac{2^{5}}{n^{2}}\left(\frac{1}{1^{6}}+\frac{1}{3^{6}}+\frac{1}{5^{6}}+\ldots\right) \frac{1}{3} e^{2} R_{\mathrm{d}}{ }^{6}\left(\frac{2 m_{\mathrm{e}}}{\hbar^{2} \pi^{2}}\right)^{2} .
$$

Further. calculations of $m_{-\tau}$, where $\tau>2$, can be readily performed. Both moments $m_{-1}$ and $m_{-2}$ contain $n^{2}$ in their denominators, so these moments are inversely proportional to the square of the quantum parameter

$$
\kappa_{n 0}=|k| \text {, }
$$

because of Eq. (46). Equation (51) holds because the energy obtained from the standing free-electron state represents the same function of the quantum parameters $\kappa_{n 0}$ as it is in the case of the dependence of the Bloch free-electron energy on the wave vector value $|k|$, see $[22,38]$.

\subsection{Relations between the moments of the strength distribution function belonging to different orders}

Traini [8] has developed several relations concerning the moments of the strength distribution function for the case of the hydrogen atom. In the present section we examine these rules in the case of the moments of the strength distribution function calculated for electrons moving in a large spherical cavity representing the metal block.

The first relation comes from the maximum condition imposed for the following combination of the moments:

$$
\int_{0}^{\infty} \mathrm{d} \omega \frac{R^{\mathrm{D}}(\omega)}{\hbar \omega}(1+\beta \hbar \omega)^{2}=m_{-1}+2 \beta m_{0}+\beta^{2} m_{1} \geq 0
$$


For the maximum value of $\beta$ which is obtained for the expression (52) it should be $[8]$

$$
m_{-1} \geq \frac{m_{0}^{2}}{m_{1}} .
$$

While examining the relation (53) we shall take into account solely the free-electron states enclosed in a spherical cavity. In this domain the electron behaviour is similar to that of free particles, so we can put

$$
\widehat{W}=\widehat{H}^{\mathrm{e}}=\frac{\hbar^{2}}{2 m_{\mathrm{e}}}\left(\widehat{p}_{x}^{2}+\widehat{p}_{y}^{2}+\widehat{p}_{z}^{2}\right) .
$$

Then, following the way demonstrated in Eqs. (7) and (27), the moment $m_{1}$ becomes

$$
m_{1}=\frac{1}{2} e^{2}\left\langle i\left|\frac{\partial^{2} \widehat{W}}{\partial \widehat{p}_{z}^{2}}\right| i\right\rangle=\frac{\hbar^{2}}{2 m_{\mathrm{e}}} e^{2} .
$$

In the next step, from the standing-like approximation for the wave functions, we obtain

$$
m_{0}=e^{2}\left\langle i\left|z^{2}\right| i\right\rangle=e^{2}\left\langle i\left|r^{2} \cos ^{2} \vartheta\right| i\right\rangle=\frac{1}{9} e^{2} R_{\mathrm{d}}^{2} .
$$

Here the spherical coordinates $r=r(r, \vartheta, \varphi)$ and the approximation

$$
\sin ^{2} \kappa r \approx \overline{\sin ^{2} \kappa r}=\frac{1}{2}
$$

have been applied for the calculations done with the aid of the free-electron wave functions extended within a spherical potential box having the radius $R_{\mathrm{d}}$. The averaging process in the expression (57) has been performed over a large interval of the variable $r$. A substitution of (49), (55) and (56) into the relation (53) gives the requirement

or

$$
\frac{2^{4}}{n^{2}} \frac{1}{3} \frac{\pi^{2}}{96} e^{2} R_{\mathrm{d}}{ }^{4} \frac{m_{\mathrm{e}}}{\hbar^{2}}>\frac{\left(1 / 3^{4}\right) e^{2} R_{\mathrm{d}}^{4}}{\hbar^{2} / 2 m_{\mathrm{e}}}
$$

$$
\frac{3^{2} \pi^{2}}{2^{2}}>n^{2}
$$

which is satisfied solely for small $n$, viz. $n=1,2,3$ and 4 .

Another relation which is fulfilled for the moments, namely

is represented by

$$
\int_{0}^{\infty} \mathrm{d} \omega \frac{R^{\mathrm{D}}(\omega)}{\hbar \omega} \leq \int_{0}^{\infty} \mathrm{d} \omega \frac{R^{\mathrm{D}}(\omega)}{\hbar \omega_{10}}
$$

$$
m_{-1} \leq \frac{m_{0}}{\hbar \omega_{10}} .
$$

For free electrons in a metal expression

$$
\hbar \omega_{10}=\frac{\hbar^{2}}{2 m_{\mathrm{e}}} \frac{\pi^{2}}{R_{\mathrm{d}}^{2}}\left[(n+1)^{2}-n^{2}\right] \approx \frac{\hbar^{2}}{m_{\mathrm{e}}} \frac{\pi^{2}}{R_{\mathrm{d}}^{2}} n
$$

represents the energy difference between two neighbouring free-electron levels. The relation $(59 \mathrm{a})$ gives the requirement

$$
\frac{2^{4}}{n^{2}} \frac{1}{3} \frac{\pi^{2}}{96} e^{2} R_{\mathrm{d}}{ }^{4} \frac{m_{\mathrm{e}}}{\hbar^{2}}<\frac{\left(1 / 3^{2}\right) e^{2} R_{\mathrm{d}}{ }^{2}}{\left(\hbar^{2} / 2 m_{\mathrm{e}}\right)\left(\pi^{2} / R_{\mathrm{d}}{ }^{2}\right) n}
$$


or

$$
\frac{\pi^{4}}{2}<n
$$

This relation is satisfied for $n \gtrsim 50$, so it holds for the overwhelming part of the free-electron states in a metal, if we note that the Fermi level of a macroscopic metal is at about $n=10^{8}$. The relation (59a) can be modified into

$$
m_{-1} \leq \frac{m_{0}}{\hbar \omega_{10}}\left[1-\frac{m_{0}}{m_{1}}\left(\hbar \omega_{10}-\frac{m_{1}}{m_{0}}\right)^{2}\left(\frac{m_{2}}{m_{1}}-\hbar \omega_{10}\right)^{-1}\right]
$$

when the inequality

$$
\int_{0}^{\infty} \mathrm{d} \omega \frac{R^{\mathrm{D}}(\omega)}{\hbar \omega}(1+\varepsilon \hbar \omega)^{2} \leq \int_{0}^{\infty} \mathrm{d} \omega \frac{R^{\mathrm{D}}(\omega)}{\hbar \omega_{10}}(1+\varepsilon \hbar \omega)^{2}
$$

is sought to be satisfied, instead of (59), for some extremal value of $\varepsilon$, see [8].

According to the formula (35a) we have for free electrons

$$
\begin{aligned}
m_{2} & =e^{2}\left\langle i\left|\left(\frac{\partial \widehat{W}}{\partial \widehat{p}_{z}}\right)^{2}\right| i\right\rangle=e^{2}\left(\frac{\hbar^{2}}{2 m_{\mathrm{e}}}\right)^{2}\left\langle i\left|\kappa^{2} \cos ^{2} \vartheta\right| i\right\rangle \\
& =e^{2}\left(\frac{\hbar^{2}}{2 m_{\mathrm{e}}}\right)^{2} \frac{n^{2} \pi^{2}}{R_{\mathrm{d}}^{2}} \frac{2}{3},
\end{aligned}
$$

where instead of the operator $\widehat{W}$ for the electron in a lattice the Hamiltonian $\hat{H}^{\mathrm{e}}$ of Eq. (54) has been applied in the first step of Eq. (63). In effect, the ratio $m_{2} / m_{1}$ is a number proportional to $n^{2}$, see Table. Evidently, for large $n$ the expression $m_{2} / m_{1}$ predominates over $\hbar \omega_{10}$ given in Eq. (60). Similarly, $\hbar \omega_{10}$ predominates . over the expression $m_{1} / m_{0}$ in Table giving

$$
\left(\hbar \omega_{10}-\frac{m_{1}}{m_{0}}\right)^{2} \approx\left(\hbar \omega_{10}\right)^{2}=\frac{\hbar^{4}}{m_{\mathrm{e}}^{2}}\left(\frac{\pi^{2}}{R_{\mathrm{d}}^{2}}\right)^{2} n^{2}
$$

for large $n$. From the relation (64) we obtain the following estimate of the corrective term within the square brackets entering the inequality (62):

$$
\begin{aligned}
& \frac{m_{0}}{m_{1}}\left(\hbar \omega_{10}-\frac{m_{1}}{m_{0}}\right)^{2}\left(\frac{m_{2}}{m_{1}}-\hbar \omega_{10}\right)^{-1} \\
& \approx \frac{2 m_{\mathrm{e}} R_{\mathrm{d}}^{2}}{9 \hbar^{2}} \frac{\hbar^{4}}{m_{\mathrm{e}}^{2}} \frac{\pi^{4}}{R_{\mathrm{d}}^{4}} n^{2} \frac{3}{2} \frac{2 m_{\mathrm{e}}}{\hbar^{2}} \frac{R_{\mathrm{d}}{ }^{2}}{n^{2} \pi^{2}}=\frac{2}{3} \pi^{2},
\end{aligned}
$$

so the relation (62) cannot be satisfied for any large $n$. It can be checked that the formula (62) cannot be satisfied also for small positive integer $n$ labelling the free-electron states.

The sequence of the ratios between the moments considered for the hydrogen atom can be arranged as follows [8]:

$$
\ldots \frac{m_{2}}{m_{1}} \geq \frac{m_{1}}{m_{0}} \geq \frac{m_{0}}{m_{-1}} \geq \frac{m_{-1}}{m_{-2}} \ldots
$$

We represent this sequence calculated for the free-electron states enclosed in a spherical cavity in Table. Only the first and the third relation presented in the formula (66) are satisfied for almost all free-electron states $n$, but none of the states $n$ can satisfy the central (second) relation entering the formula (66). 
TABLE

Sequence of the moments ratios calculated for the free-electron states enclosed in a spherical cavity of the radius $R_{\mathrm{d}}$. The free-electron energy in state $n$ in the cavity is equal to $E_{n}=\frac{\hbar^{2}}{2 m_{c}} \frac{n^{2} \pi^{2}}{R_{d}^{2}}$ on condition that the electron states labelled by $l=m=0$ are taken into consideration, see Eq. (40).

$$
\begin{aligned}
& \frac{m_{2}}{m_{1}}=\frac{e^{2}\left(\frac{\hbar}{2 m_{e}}\right)^{2} \frac{n^{2} \pi^{2}}{R_{\mathrm{d}}^{2}} \frac{2}{3}}{\frac{\hbar^{2}}{2 m_{\mathrm{e}}} e^{2}}=\frac{2}{3} \frac{\hbar}{2 m_{\mathrm{e}}} \frac{n^{2} \pi^{2}}{R_{\mathrm{d}}^{2}}=\frac{2}{3} E_{n} \\
& \frac{m_{1}}{m_{0}}=\frac{\frac{\hbar^{2}}{2 m_{\mathrm{e}}} e^{2}}{\frac{1}{9} e^{2} R_{\mathrm{d}}^{2}}=\frac{1}{9 \pi^{2}} \frac{\hbar^{2}}{2 m_{\mathrm{e}}} \frac{\pi^{2}}{R_{\mathrm{d}}^{2}}=\frac{1}{9 \pi^{2}} E_{1} \\
& \frac{m_{0}}{m_{-1}}=\frac{\frac{1}{9} e^{2} R_{\mathrm{d}}^{2}}{\frac{2^{4}}{\mathrm{n}^{2}} \frac{1}{3} \frac{\pi^{2}}{96} \mathrm{e}^{2} R_{\mathrm{d}}^{4} \frac{m_{\mathrm{e}}}{\bar{\hbar}^{2}}}=\frac{4}{\pi^{4}} \frac{\hbar^{2}}{2 m_{\mathrm{e}}} \frac{\pi^{2} n^{2}}{R_{\mathrm{d}}^{2}}=\frac{4}{\pi^{4}} E_{n} \\
& \frac{m_{-1}}{m_{-2}}=\frac{\frac{1}{6 n^{2}} e^{2} R_{\mathrm{d}}^{4} \frac{m_{e}}{\hbar^{2}}}{4 \frac{\pi^{4}}{15 n^{2}} e^{2} R_{\mathrm{d}}^{6}\left(\frac{m_{\mathrm{e}}}{\hbar^{2} \pi^{2}}\right)^{2}}=\frac{15}{12} \frac{\hbar^{2}}{m_{\mathrm{e}}} \frac{1}{R_{\mathrm{d}}^{2}}=\frac{5}{2 \pi^{2}} E_{1}
\end{aligned}
$$

\section{Discussion of results obtained in Sec. 6}

The $m_{1}$-sum rules obtained in the present paper for cubic lattices (Sec. 5) can be developed also for other lattices. The intermediate situations between free electrons and tightly-bound electrons can be equally considered. Any of such situations, representing a definite strength of the atomic interaction in a lattice, is characterized by a different energy operator $\widehat{W}$ acting on the LCAO coefficient functions.

A calculation of the moments $m_{-\tau}$ having negative indices $(\tau>0)$ has been done for free electrons (see Sec. 6) and a question may arise how the crystal lattice can influence the free-electron results obtained for $m_{-\tau}$. An answer can be attained if the free-electron states are replaced by the nearly free-electron states in cubic lattices. In the second case the standing LCAO-wave functions representing a band of nearly-free $s$-electrons moving in the field of a cubic crystal lattice are applied. For nearly-free electrons, as well as electrons close to the band limit, the coefficient functions do not depend on the kind of the cubic lattice [21]; we assumed here that the situation at the band limit coincides with the electron states having small values of the quantum parameter $\kappa$. The volume of the lattice is limited to a spherical block the centre of which is located in one of the sites of the crystal lattice.

In general, the diagonalization procedure is applied to the Wannier-Slater energy eigenequation (22) and this is done for any cubic lattice. The procedure 
splits the original free-electron band of states into subbands labelled by the index $\lambda$ and the index $\Gamma$ labels the irreducible representation of the cubic point group, see Eq. (31). The energy expression calculated for each subband $\lambda$ depends on the quantum parameter $\kappa$ in the following way:

$$
E^{c}=E^{\Gamma, \lambda}(\kappa)=a_{0}+a_{1} \kappa^{2}+a_{2}^{\Gamma, \lambda} \kappa^{4}+a_{3}^{\Gamma, \lambda} \kappa^{6}+\ldots
$$

the superscript $\Gamma$ and $\lambda$ have been omitted for $a_{0}$ and $a_{1}$ since these coefficients do not depend on $\Gamma$ and $\lambda$. The corresponding eigenfunctions of Eq. (22) are

$$
A(\boldsymbol{R}) \equiv A^{\Gamma, \mu, \lambda}(\kappa, \boldsymbol{R})=\sum_{l} \sum_{t} c_{l, t}^{\Gamma, \mu, \lambda}(\kappa)(H)_{l, t}^{\Gamma, \mu} j_{l}(\kappa R)
$$

where $(H)_{i, t}^{\Gamma}$ are the lattice (cubic) harmonics belonging to a certain specified irreducible representation $\Gamma$ whereas the index $\mu$ labels the rows of $\Gamma$. The coefficients $c_{l, t}^{\Gamma, \mu, \lambda}(\kappa)$ in Eq. (68), as well as $a_{i}^{\Gamma, \lambda}$ in Eq. (67), are obtained in the course of the diagonalization process done for a large crystal block. Since for a macroscopic crystal block a difference between two neighbouring values of the quantum parameter $\kappa$ can be a very small number, a small energy difference entering the denominator of $m_{-1}$ and $m_{-2}$ can be attained mainly for two neighbouring $\kappa$, say $\kappa^{\prime}$ and $\kappa^{\prime \prime}$, entering the same $E^{\Gamma_{i}, \lambda}(\kappa)$. In this way the energy difference coming from the electron states belonging to different $\lambda$ can be neglected and the expression entering the denominator of $m_{-1}$ and $m_{-2}$ is reduced solely to the difference

$$
E^{\Gamma, \lambda}\left(\kappa^{\prime}\right)-E^{\Gamma, \lambda}\left(\kappa^{\prime \prime}\right) \cong a_{1}\left(\kappa^{\prime 2}-\kappa^{\prime \prime 2}\right),
$$

which is an expression typical of the free-electron states. This result holds for all $\Gamma$ and $\lambda$. Moreover, the coefficient functions (68) having different $\lambda$ belong, for a given energy, to evidently different $\kappa$, so these functions strongly interfere in the matrix element

$$
\left\langle A^{\Gamma_{i}, \mu_{i}, \lambda^{\prime}}\left(\kappa^{\prime}, R\right)|Z| A^{\Gamma_{j}, \mu_{j}, \lambda^{\prime \prime}}\left(\kappa^{\prime \prime}, R\right)\right\rangle
$$

calculated for $\Gamma_{i} \neq \Gamma_{j}$. An exception is the situation considered at the band limit where $\kappa^{\prime} \approx \kappa^{\prime \prime}$ holds also for the case of $\lambda^{\prime} \neq \lambda^{\prime \prime}$, and this situation is chosen in a further examination.

First let us note that the direct product between representations $\Gamma_{i}, \Gamma_{j}$ and representation $\Gamma_{15}$ to which belongs the variable $Z$ entering the formula (70) should contain the total-symmetry representation $\Gamma_{1}$, otherwise the matrix element (70) vanishes, for the transformation properties of the basis functions calculated for different $\Gamma_{i}$ see e.g. $[40,41]$. If the lattice site

$$
\boldsymbol{R}=\mathbf{0}
$$

is chosen as a typical site of all lattice sites of the crystal block, then solely the matrix elements coming from

$$
\Gamma_{i}=\Gamma_{1}, \quad \Gamma_{j}=\Gamma_{15},
$$

or vice versa, should be considered in the formula (70). In the case of the relations (72) the index $\mu$ for the representation $\Gamma_{1}$ does not enter into play (see e.g. [40]) and $\mu_{j}=Z$ because solely this row index can provide us with a non-vanishing result in the formula (70). 
A sum of the matrix elements (70) done for different sets of $\lambda^{\prime}$ and $\lambda^{\prime \prime}$ has been performed in Ref. [42], where also the standing-like LCAO coefficient functions of the $s$ electrons in cubic lattices have been presented; cf. here also [21-25]. For the band limit, the coefficient functions $A^{\Gamma_{i}, \mu_{i}, \lambda^{\prime}}\left(\kappa^{\prime}, \boldsymbol{R}\right)$ and $A^{\Gamma_{j}, \mu_{j}, \lambda^{\prime \prime}}\left(\kappa^{\prime \prime}, \boldsymbol{R}\right)$ degenerate to expressions corresponding to the small values of parameters $\kappa^{\prime}$ and $\kappa^{\prime \prime}$, Then the following relation is satisfied for small $\kappa^{\prime} \approx \kappa_{n 0}$ and $\kappa^{\prime \prime} \approx \kappa_{n^{\prime} 10}$, so this relation is practically the same for all kinds of cubic lattices

$$
\begin{aligned}
& \sum_{\mu^{\prime}} \sum_{\mu^{\prime \prime}} \sum_{\lambda^{\prime}} \sum_{\lambda^{\prime \prime}} w^{\Gamma_{1}, \lambda^{\prime}}\left(\kappa^{\prime}\right) w^{\Gamma_{15}, Z, \lambda^{\prime \prime}}\left(\kappa^{\prime \prime}\right)\left\langle\Gamma_{1}, \lambda^{\prime}|Z| \Gamma_{15}, Z, \lambda^{\prime \prime}\right\rangle \\
& \quad=\left\langle\kappa^{\prime}, 0,0|Z| \kappa^{\prime \prime}, 1,0\right\rangle
\end{aligned}
$$

where

$$
\begin{aligned}
& \left|\Gamma_{1}, \lambda^{\prime}\right\rangle=\frac{A^{\Gamma_{1}, \lambda^{\prime}}}{\left[w^{\Gamma_{1}, \lambda^{\prime}}(\kappa)\right]^{1 / 2}}=\frac{\kappa v_{\mathrm{a}}^{1 / 2}}{\left(2 \pi R_{\mathrm{d}}\right)^{1 / 2}}\left[(H)_{0}^{\Gamma_{1}}+c_{4}^{\Gamma_{1}, \lambda^{\prime}}\left(\kappa^{\prime}\right)(H)_{4}^{\Gamma_{1}}\right. \\
& \left.-c_{6}^{\Gamma_{1}, \lambda^{\prime}}\left(\kappa^{\prime}\right)(H)_{6}^{\Gamma_{1}}+c_{8}^{\Gamma_{1}, \lambda^{\prime \prime}}\left(\kappa^{\prime}\right)(H)_{8}^{\Gamma_{1}}+\ldots\right] \frac{\sin \left(\kappa^{\prime} R\right)}{\kappa^{\prime} R} \\
& \left|\Gamma_{15}, Z, \lambda^{\prime \prime}\right\rangle=\frac{A^{\Gamma_{15}, Z, \lambda^{\prime \prime}}}{\left[w^{\Gamma_{15}, Z, \lambda^{\prime \prime}}(\kappa)\right]^{1 / 2}}=\frac{3^{1 / 2} \kappa^{\prime \prime} v_{\mathrm{a}}^{1 / 2}}{\left(2 \pi R_{\mathrm{d}}\right)^{1 / 2}}\left[(H)_{1}^{\Gamma_{15}, Z}-c_{3}^{\Gamma_{15}, z, \lambda^{\prime \prime}}(H)_{3}^{\Gamma_{15}, Z}\right. \\
& \left.+c_{5,1}^{\Gamma_{15}, Z, \lambda^{\prime \prime}}\left(\kappa^{\prime \prime}\right)(H)_{5,1}^{\Gamma_{15}, Z}+c_{5,2}^{\Gamma_{15}, Z, \lambda^{\prime \prime}}\left(\kappa^{\prime \prime}\right)(H)_{5,2}^{\Gamma_{15}, Z}+\ldots\right]\left[-\frac{\cos \left(\kappa^{\prime \prime} R\right)}{\kappa^{\prime \prime} R}\right] \\
& {\left[w^{\Gamma_{1}, \lambda^{\prime}}(\kappa)\right]^{2}=\left\{1+\frac{16}{525}\left[c_{4}^{\Gamma_{1}, \lambda^{\prime}}\left(\kappa^{\prime}\right)\right]^{2}+\frac{32}{77077}\left[c_{6}^{\Gamma_{1}, \lambda^{\prime}}\left(\kappa^{\prime}\right)\right]^{2}\right.} \\
& \left.+\frac{256}{2370225}\left[c_{8}^{\Gamma_{1}, \lambda^{\prime}}\left(\kappa^{\prime}\right)\right]^{2}+\ldots\right\}^{-1} \\
& {\left[w^{\Gamma_{15}, Z, \lambda^{\prime \prime}}(\kappa)\right]^{2}=\left\{1+\frac{12}{175}\left[c_{3}^{\Gamma_{15}, Z, \lambda^{\prime \prime}}\left(\kappa^{\prime \prime}\right)\right]^{2}+\frac{64}{14553}\left[c_{5,1}^{\Gamma_{15}, Z, \lambda^{\prime \prime}}\left(\kappa^{\prime \prime}\right)\right]^{2}\right.} \\
& \left.+\frac{4}{1155}\left[c_{5,2}^{\Gamma_{1,5}, Z, \lambda^{\prime}}\left(\kappa^{\prime \prime}\right)\right]^{2}+\ldots\right\}^{-1}, \\
& \left|\kappa^{\prime}, 0,0\right\rangle=N_{00}^{\Gamma_{1}}(H)_{0}^{\Gamma_{1}} j_{0}\left(\kappa^{\prime} R\right)=\frac{\kappa^{\prime}}{(2 \pi R)^{1 / 2}} \frac{\sin \left(\kappa^{\prime} R\right)}{\kappa^{\prime} R} \\
& (75 \mathrm{a})
\end{aligned}
$$

In Eqs. (74), (74a) and (76), (76a) the asymptotic formulae valid at large $R$ are taken into account for the spherical Bessel functions $j_{l}$ belonging to different $l$; 
see [39]. For even $l$ these formulae become equal to the expression of $j_{0}\left(\kappa^{\prime}, R\right)$ and for odd $l$ the asymptotic formulae become equal to the asymptotic expression of $j\left(\kappa^{\prime \prime}, R\right)$ on condition that a proper sign is taken before $j_{0}\left(\kappa^{\prime}, R\right)$ [see $(40)$ ] and before the asymptotic form of $j_{1}\left(\kappa^{\prime \prime}, R\right)$ which becomes

$$
j_{1}\left(\kappa_{n^{\prime} 1} r\right) \approx-\frac{\cos \left(\kappa_{n^{\prime} 1} r\right)}{\kappa_{n^{\prime} 1} r}
$$

[see Eq. (41)]; in Eq. (40) the parameter $\kappa^{\prime}$ is replaced by $\kappa_{n 0}$ and in the relations (41) and (77) $\kappa^{\prime \prime}$ is replaced by $\kappa_{n^{\prime} 1}$. A notation characteristic of the symmetry species of the cubic point group has been introduced into Eqs. (76) and (76a); the volume $v_{\mathrm{a}}$ occupied by a single atom in the lattice has been put equal to 1 .

The result obtained in Eq. (75) - together with that given in the relation (69) - implies that the free-electron calculation of the moments $m_{-1}, m_{-2}, \ldots$ are justified also for electrons moving in the field of the solid lattice on condition that the quantum parameters $\kappa^{\prime}$ and $\kappa^{\prime \prime}$ are small, so they correspond to the values $\left|k^{\prime}\right|$ and $\left|k^{\prime \prime}\right|$ of the wave vectors being not far from the centre of the first Brillouin zone, see [22].

\section{References}

[1] H.A. Bethe, R. Jackiw, Intermediate Quantum Mechanics, W.A. Benjamin, New York 1968.

[2] H.A. Bethe, E.E. Salpeter, The Quantum Mechanics of One- and Two-Electron Atoms, Plenum, New York 1977.

[3] J.C. Slater, Quantum Theory of Atomic Structure, Vol. 2, McGraw-Hill, New York 1960.

[4] W. Thomas, Naturwissenschaften 13, 627 (1925).

[5] F. Reiche, W. Thomas, Z. Phys. 34, 510 (1925).

[6] W. Kuhn, Z. Phys. 33, 408 (1925).

[7] E. Merzbacher, Quantum Mechanics, Wiley, New York 1970.

[8] M. Traini, Europ. J. Phys. 17, 30 (1996).

[9] U. Fano, J.W. Cooper, Rev. Mod. Phys. 40, 441 (1968).

[10] C. Baxter, Phys. Rev. A 50, 875 (1994).

[11] R. Jackiw, Phys. Rev. 157, 1220 (1967).

[12] S. Stringari, Phys. Rev. B 46, 2974 (1992).

[13] F. Dalfovo, S. Stringari, Phys. Rev. B 46, 13991 (1992).

[14] O. Madelung, Introduction to Solid-State Theory, Springer, Berlin 1978.

[15] J.M. Ziman, Principles of the Theory of Solids, University Press, Cambridge 1972.

[16] F. Bloch, Z. Phys. 52, 555 (1928).

[17] J. Reitz, in: Solid State Physics, Eds. F. Seitz, D. Turnbull, Vol. 1, Academic, New York 1955, p. 1.

[18] G.H. Wannier, Phys. Rev. 52, 191 (1937).

[19] J.C. Slater, Phys. Rev. 76, 1592 (1949).

[20] J.C. Slater, Quantum Theory of Molecules and Solids, Vol. 3, McGraw-Hill, New York 1967.

[21] S. Olszewski, Phys. Rev. B 3, 4361 (1971). 
[22] P. Modrak, S. Olszewski, Phys. Rev. B 14, 2387 (1976).

[23] T. Bulski, P. Modrak, S. Olszewski, Acta Phys. Pol. A 60, 183 (1981).

[24] A. Wierzbicki, Acta Phys. Pol. A 41, 67 (1972); ibid. 42, 37 (1972).

[25] A. Kunert, Int. J. Quantum Chem. 14, 127 (1978).

[26] A. Holas, S. Olszewski, A. Wierzbicki, Phys. Rev. B 32, 7890 (1985).

[27] G.H. Wannier, Rev. Mod. Phys. 34, 645 (1962).

[28] J. Friedel, in: Optical Properties of Solids, Ed. F. Abelès, North-Holland, Amsterdam 1972, p. 1.

[29] D. Brzeska, S. Olszewski, Int. J. Quantum Chem. 45, 105 (1993).

[30] V.M. Genkin, P.M. Mednis, Zh. Eksp. Teor. Fiz. 54, 1137 (1968) [Sov. Phys. JETP 27, 609 (1968)].

[31] J. Callaway, Quantum Theory of the Solid State, Part B, Academic, New York 1974.

[32] E. Hadjimichael, W. Currie, S. Fallieros, Am. J. Phys. 65, 335 (1997).

[33] J.C. Slater, Quantum Theory of Atomic Structure, Vol. 1, McGraw-Hill, New York 1960.

[34] S. Olszewski, A. Wierzbicki, Phys. Status Solidi B 65, 779 (1974).

[35] J.C. Slater, G.F. Koster, Phys. Rev. 94, 1498 (1954).

[36] G.C. Cho, T. Dekorsy, H.J. Bakker, R. Hövel, H. Kurz, Phys. Rev. Lett. 77, 4062 (1996).

[37] R.I. Liboff, Phys. Rev. A 43, 5765 (1991).

[38] S. Olszewski, T. Roliński, T. Kwiatkowski, Proc. R. Soc. Lond. A 453, 987 (1997).

[39] A. Gray, G.B. Mathews, A Treatise on Bessel Functions and their Applications to Physics, MacMillan, London 1952.

[40] J. Callaway, Quantum Theory of the Solid State, Part A, Academic, New York 1974.

[41] S.L. Altmannn, A.P. Cracknell, Rev. Mod. Phys. 37, 19 (1965).

[42] S. Olszewski, T. Kwiatkowski, Int. J. Quantum Chem. 57, 113 (1996). 